\title{
Naming Game Dynamics on Pairs of Connected Networks with Competing Opinions
}

\author{
Albert Trias Mansilla ${ }^{1}$, Mingming Chen ${ }^{2}$, Boleslaw K. Szymanski ${ }^{2,3}$ \\ and Josep Lluís de la Rosa Esteva ${ }^{1}$ \\ ${ }^{1}$ EASY Innovation Center, University of Girona, Girona, Spain \\ \{albert.trias, joseplluis.delarosa\}@udg.edu \\ ${ }^{2}$ Network Science and Technology Center, RPI, Troy, NY, USA \\ \{chenm8, szymab\}@rpi.edu \\ ${ }^{3}$ Computer Science and Management, Wroclaw University of Technology, Wroclaw, Poland
}

\begin{abstract}
We study the Naming Game (NG) dynamics when two disjoint networks with nodes in consensus on competing opinions are connected with new links. We consider two sets of networks; one contains several networks with real-life communities, the other networks generated with the Watts-Strogatz and Barabási-Albert models. For each set, we run NG on all the possible pairs of networks and observe whether a consensus is reached to determine network features that correlate highly with such outcome. The main conclusion is that the quality of network community structure informs network's ability to resist or exert influence from/on others. Moreover, the outcomes depend on whether Speaker-First of Listener-First NG is run and on whether a speaker or listener is biased towards high or low degree nodes. The results reveal strategies that may be used to enable and accelerate convergence to consensus in social networks.
\end{abstract}

Keywords: Social Networks · Social Influence $\cdot$ Naming Game $\cdot$ Simulations

\section{Introduction}

Research on opinion dynamics [1,2] has been concerned with the effect of social influence. Prior research has shown that the interpersonal relationships often drive large-scale changes to the opinions in the network and thus have a dominant effect on opinion adoption and spread [3]. Several models have been proposed to incorporate this effect such as the Voter Model [4], Threshold Model [5], Bass Model [6], and the Naming Game (NG) [7,8]. Unlike the other models, the Naming Game allows each node to possess more than one opinion at a time. This paper focuses on the Naming Game.

The model consists of a social network of $|\mathrm{V}|$ agents where each of these agents has a list of opinions. In each step of the Naming Game, there are $O(|V|)$ pairwise communications between two randomly selected agents, one of which plays the role of speaker and the other the role of listener. The speaker transmits to the listener an opinion from its list. If the opinion sent is on the listener's list then both agents reduce their list to a single opinion sent by the speaker. Otherwise, the listener adds the opin-

adfa, p. 1, 2011.

(C) Springer-Verlag Berlin Heidelberg 2011 
ion sent to its list. The order in which speaker and listener are selected has a strong impact on the model. Choosing the listener first increases the chance for selecting a speaker with the degree higher than average. Consequently, in scale free networks, hubs will be the most frequent speakers, giving rise to faster convergence to consensus [9].

Empirical social graphs exhibit strong community structure that inhibits achieving consensus. Methods and conditions for achieving it in social networks have been studied and included use of committed agents [8] or global external signals [10, 11].

In this paper, we study the outcomes of Naming Game in which two disconnected networks with nodes in consensus on competing opinions are joined by newly added links. We study which network has higher chance to converge the joined network to its opinion. Consequently, we focus on the so-called Binary Naming Game, in which only two opinions, $\mathrm{A}$ and $\mathrm{B}$, and their union, $\mathrm{AB}$, can appear on the list of opinions of any agent.

\section{Network Properties and Community Quality}

The most basic properties of a network are the number of nodes $(|\mathrm{V}|)$ and the number of edges $(|E|)$. The assortative coefficient $(r)$ measures the tendency of a node connecting to other nodes with similar degrees. A positive $r$ indicates that most connections exist between nodes with similar degrees (assortative network), while a negative $r$ implies a disassortative network which is often more vulnerable to random failures and targeted attacks [12]. The diameter (d) defines the longest shortest path in a network, so it is representative of the linear size of the network. Finally, the spectrum that contains the eigenvalues of the adjacency matrix is an important characteristic, especially the largest eigenvalue $(\lambda)$, where a higher value of $\lambda$ means a smaller diameter and more robustness of the network [12].

Among community quality metrics, modularity [13] is widely used to effectively measure the strength of the community structure found by community detection algorithms. This metric measures the difference between the fraction of all edges that are within the actual community and such a fraction of edges that would be inside the community in a randomized graph with the same number of nodes and the same degree sequence. For the given community partition of a network $G=(V, E)$ with $|E|$ edges and $|\mathrm{V}|$ nodes, modularity $(\mathrm{Q})$ is given by

$$
Q=\sum_{c \in C}\left[\frac{\left|E_{c}^{\text {in }}\right|}{|E|}-\left(\frac{2\left|E_{c}^{\text {in }}\right|+\left|E_{c}^{\text {out }}\right|}{2|E|}\right)^{2}\right],
$$

where $C$ is the set of all the communities, $\left|E_{c}^{i n}\right|$ is the number of edges between nodes within the specific community c, and $\left|E_{c}^{\text {out }}\right|$ is the number of edges from the nodes in community c to the nodes outside c.

To address well-known modularity shortcomings, Chen et al. [14] propose to modify modularity by subtracting from it the fraction of edges connecting nodes of different communities and by including community density in formula. The resulting metric, called modularity density $\left(\mathrm{Q}_{\mathrm{ds}}\right)$, is defined as 


$$
\begin{aligned}
& Q_{d s}=\sum_{c \in C}\left[\frac{\left|E_{c}^{\text {in }}\right|}{|E|} d_{c}-\left(\frac{2\left|E_{c}^{\text {in }}\right|+\left|E_{c}^{\text {out }}\right|}{2|E|} d_{c}\right)^{2}-\sum_{\substack{c^{\prime} \in C \\
c^{\prime} \neq c}} \frac{\left|E_{c, c^{\prime}}\right|}{2|E|} d_{c, c^{\prime}}\right], \\
& d_{c}=\frac{2\left|E_{c}^{\text {in }}\right|}{|c|(|c|-1)}, \quad d_{c, c^{\prime}}=\frac{\left|E_{c, c^{\prime}}\right|}{\left|c \| c^{\prime}\right|} .
\end{aligned}
$$

In the above, $|c|$ is the size of community c, $d_{c}$ is the internal density of community $c$, and $d_{c, c^{\prime}}$ is the pair-wise density between communities $\mathrm{c}$ and $\mathrm{c}^{\prime}$.

We also use the following community quality metrics.

The number of Intra-edges $\left|E_{c}^{i n}\right|$ is the total number of edges in the community c and it tends to be high for communities of high quality.

Intra-density, $d_{c}$ in the definition of $Q_{d s}$, tends to be high for high quality communities.

Contraction $2\left|E_{c}^{i n}\right| /|c|$ measures the average node intra-edge degree for edges internal to the community and it tends to be high for communities of high quality.

The number of Boundary-edges $\left|E_{c}^{\text {out }}\right|$ that connect nodes in community c to nodes outside $\mathrm{c}$ and it tends to be low for high quality communities.

Expansion $\left|E_{c}^{\text {out }}\right| /|c|$ measures the average inter-edge node degree for edges that connect to nodes outside the community c; it tends to be low for high quality communities.

Inter-density $\left|E_{c}^{\text {out }}\right| /(|c|(|V|-|c|))$ measures the inter-edge density and it tends to be low for communities with high quality.

Conductance $\frac{\left|E_{c}^{\text {out }}\right|}{2\left|E_{c}^{\text {in }}\right|+\left|E_{c}^{\text {out }}\right|}$ measures the fraction of edges that point outside community c; it tends to be low for communities of high quality.

Additionally, we also considered the number of communities $(|\mathrm{C}|)$ and the largest (highest value of) size of community (HSC) in the network.

\section{$3 \quad$ Naming Game On Pairs of Networks}

We study the Naming Game dynamics on pairs of networks to analyze what properties of a network define its resilience to external opinions and its ability to impose its opinion on other networks. Like in [15], we start with two disjoint networks, each with nodes in consensus on opinion different from consensus opinion in the other network. Then, we introduce links from one network to the other, modeling the response to incentives for forming new links between agents from different networks. After the NG stabilizes the opinion distribution, we record the outcome. Since our goal is to observe under what conditions the joint network reaches consensus, we discard from our results cases in which no consensus on a single opinion is reached.

We investigate the following research questions: What are the network properties that influence the outcome? How does the outcome depend on the type of Naming Game and the selection of a partner for opinion exchange? In term of influence, answers to these questions may help to identify sub-networks which are good influenc- 
ers, or, opposite, which are easy to be influenced. These answers may also be useful in designing strategies for increasing the selected sub-networks resilience to influence or their ability to influence other sub-networks.

It is clear [15] that too small number of added links will not yield the consensus and very large number of links will benefit the network with the larger number of nodes. The interesting region is right in middle of these two extremes, when the outcome depends on structural properties of networks and their communities. For simplicity and brevity sake, in this paper, we only investigate the addition of a constant number of links (100), but the sizes of the two joined networks vary in our analyses, thus, also the percentage of added links does.

\subsection{An Example of NG Dynamics on a Pair of Networks}

Given are two networks with four nodes each. The network A has a ring topology (4Ring) while network B is fully connected (4All). All nodes in network A have opinion $\mathrm{A}$, while in network $\mathrm{B}$ they all have opinion $\mathrm{B}$. We connect both networks by a single link, and for simplicity of the analysis, change the opinions of nodes connected by this link to a mixed opinion $\{\mathrm{A}, \mathrm{B}\}$. The start of the scenario is shown in Fig. 1.

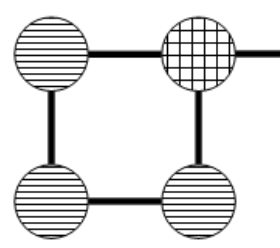

Network A

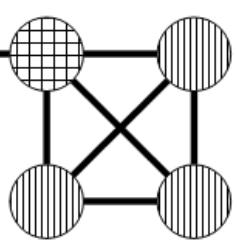

Network B

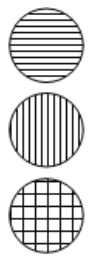

\# A $A$ and $B$

Fig. 1. The start of scenario

For the Speaker First Naming Game (SF-NG), a straightforward analysis (omitted here for the sake of the brevity) shows that both networks have a same probability, $1 / 8$ of removing the outsiders' opinion from their nodes. However, the probability of propagating the outsiders' opinion inside each network is 3/64 for opinion A and 1/24 for opinion B. Thus, for networks with the comparable number of nodes, the one that has weaker community structure or fewer links has higher probability of propagating its consensus opinion to the other network and, at the same time, it has lower probability of getting the outsiders' opinion propagated to its own nodes.

Similar elementary analysis for the Listener First Naming Game (LF-NG) case shows that both networks have the same probability of propagating the outsiders' opinion inside each network. But network B is more likely than network A to resist permanent holding of the outsiders' opinion by its nodes. Here, the conclusion is reversed compared to the previous case and the network with more links or stronger community structure is favored to impose consensus to its opinion in LF-NG. 
The simulation results corresponding to the discussed cases are shown in Tables 1 and 2.

Table 1. Percentage of games that network A wins over network B in the SF-NG followed by the difference in the number of links and by the difference in their intra-densities.

$\begin{array}{lrrrrr}\text { A/B } & \text { 3 } & \text { 4Ring } & \text { 4All } & \text { 5Ring } & \text { 5All } \\ \text { 3 } & 0.5 ; 0 ; 0 & 0.40 ;-1 ; 0.25 & 0.47 ;-3 ; 0 & 0.33 ;-1 ; 0.5 & 0.43 ;-7 ; 0 \\ \text { 4Ring } & 0.61 ; 1 ;-0.25 & 0.5 ; 0 ; 0 & 0.56 ;-2 ;-0.25 & 0.43 ;-1 ; 0.25 & 0.55 ;-6 ;-0.25 \\ \text { 4All } & 0.52 ; 1 ;-0.25 & 0.43 ; 2 ; 0.25 & 0.5 ; 0 ; 0 & 0.35 ; 1 ; 0.5 & 0.47 ;-4 ; 0 ; \\ \text { 5Ring } & 0.67 ; 2 ;-0.5 & 0.57 ; 1 ;-0.25 & 0.65 ;-1 ;-0.5 & 0.5 ; 0 ; 0 & 0.61 ;-5 ;-0.5 \\ \text { 5All } & 0.573 ; 7 ; 0 & 0.46 ; 6 ; 0.25 & 0.54 ; 4 ; 1 & 0.40 ; 5 ; 0.5 & 0.5 ; 0 ; 0\end{array}$

Table 2. Percentage of games that network A wins network B in the LF-NG, followed by the difference in the number of links and by the difference in intra-densities.

$\begin{array}{lrrrrr}\text { A } \backslash \mathrm{B} & \mathbf{3} & \text { 4Ring } & \text { 4All } & \mathbf{5 R i n g} & \text { 5All } \\ \text { 3 } & 0.5 ; 0 ; 0 & 0.42 ;-1 ; 0.25 & 0.30 ;-3 ; 0 & 0.37 ;-1 ; 0.5 & 0.17 ;-7 ; 0 \\ \text { 4Ring } & 0.57 ; 1 ;-0.25 & 0.5 ; 0 ; 0 & 0.36 ;-2 ;-0.25 & 0.44 ;-1 ; 0.25 & 0.23 ;-6 ;-0.25 \\ \text { 4All } & 0.706 ; 3 ; 0 & 0.63 ; 2 ; 0.25 & 0.5 ; 0 ; 0 & 0.56 ; 1 ; 0.5 & 0.33 ;-4 ; 0 \\ \text { 5Ring } & 0.64 ; 2 ;-0.5 & 0.56 ; 1 ;-0.25 & 0.42 ;-1 ;-0.5 & 0.5 ; 0 ; 0 & 0.27 ;-5 ;-0.5 \\ \text { 5All } & 0.84 ; 7 ; 0 & 0.78 ; 6 ; 0.25 & 0.68 ; 4 ; 1 & 0.73 ; 5 ; 0.5 & 0.5 ; 0 ; 0\end{array}$

Tables 1 and 2 show high correlation between the difference of the number of links and the percentage of games that the network A wins network B with LF-NG. There is also a high inverse correlation between the difference in intra-densities of the networks and the percentage of wins with the SF-NG. When the intra-densities are the same, the network with more nodes has advantage.

\section{$4 \quad$ Method}

23 networks were generated using the Watts-Strogatz and Barabási-Albert models. Then, we used the fine-tuned $\mathrm{Q}_{\mathrm{ds}}$ algorithm to detect the community structures of these networks [16]. Finally, we calculated the values of the community quality metrics on the discovered community structure of each generated network.

In terms of properties, the sizes of generated networks ranged from 100 to 1000 nodes and from 191 to 7,000 edges. The other parameters ranged as follows: the number of communities, $|C| \in[1,104]$, the assortative coefficient $r \in[-0.1772$, 0.2697], the network diameter $d \epsilon[3,38]$, the modularity density $Q_{d s} \in[0.039,0.471]$, and the modularity $\mathrm{Q} \in[0,0.684]$. The quality metrics have the following ranges: intra-density, ID [0.16, 0.788], conductance CND $€[0,0.788]$, expansion EXP $€[0$, $5.991]$, border density $\mathrm{BD} \in[0,0.04]$, the number of boundary-edges $\mathrm{BE} \in[0$, 76.789], contraction CNT [1.418, 15.84], the number of intra-edges IE $€$ [2.904, 1175.67]. In addition we measured the number of converged games over 100 Naming 
Game runs, CG $\epsilon[41,100]$, and the mean number of steps, $s$, that were needed to bring the game to consensus ( $\mathrm{s} \in[20,3618])$.

Then, we selected all possible pairs of all networks for the games. Each pair was connected with $\Delta \mathrm{L}=100$ new links between nodes of different networks. Both SF-NG and LF-NG were run 5000 times with different random generator seed for each configuration for up to $\mathrm{M}=5,000$ steps. The executed algorithm is sketched in Table 3 . We used three strategies for selecting the second agent for each conversation (speaker or listener) in each case. The strategies are selected by parameter $x=\{0,1,-1\}$ that defines probability of selecting the node $i$ with the degree $n_{i}$ is as follows:

$$
p(i)=\frac{n_{i}{ }^{x}}{\sum_{j=1}^{|V|} n_{j}^{x}} .
$$

Thus, strategy with $x=0$ is the usual one, where all the neighbors have the same probability of being chosen. For strategy with $\mathrm{x}=1$, the selection is biased towards high degree nodes while the opposite bias arises for strategy with for $\mathrm{x}=-1$.

Table 3. Implemented NG Algorithm

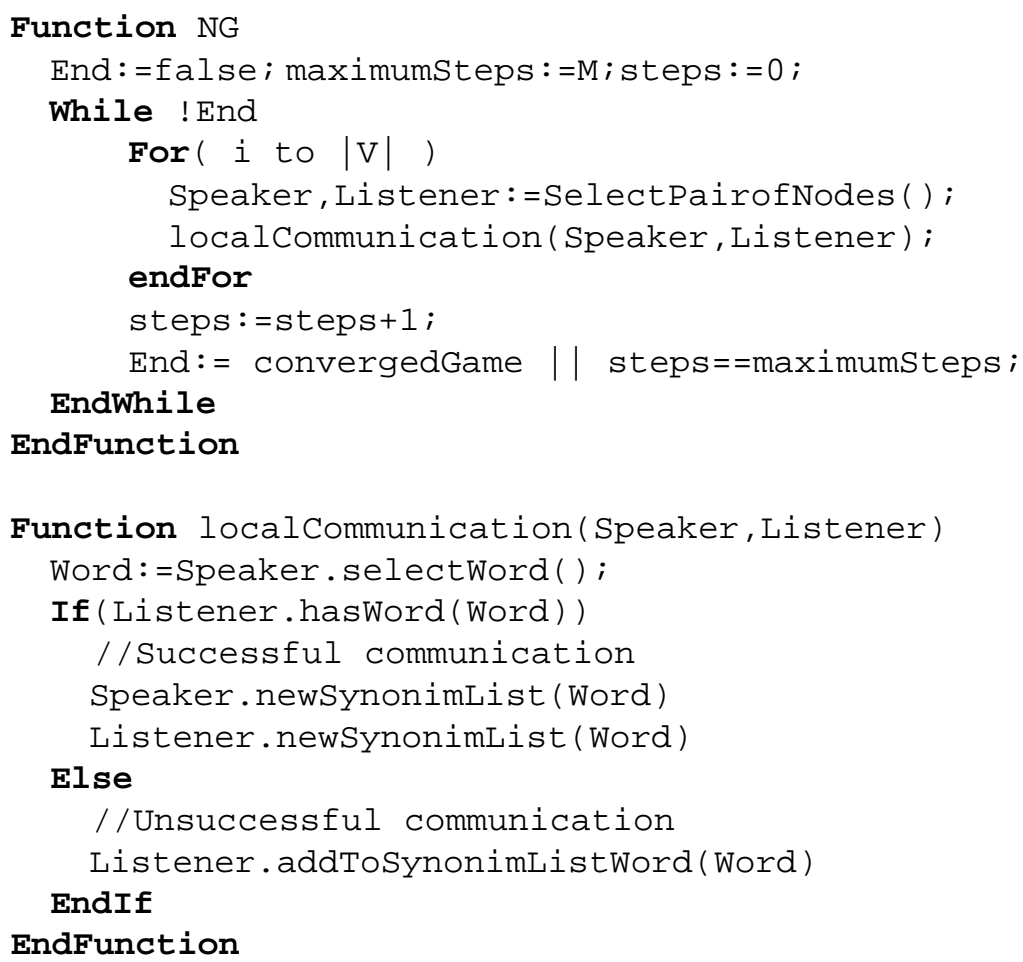

We collected the following results.

$W_{i, j} \in[0,1], D_{i, j} \in[0,1], L_{i, j} \in[0,1], S_{i, j} \in[-1,1]$ indicate, respectively, the fraction of wins, draws, losses, and the fraction of wins less the fraction of losses over the converged games between networks $\mathrm{c}_{\mathrm{i}}$ and $\mathrm{c}_{\mathrm{j}}$. However, only $\mathrm{S}$ was used in analysis. 
Each input variable is the ratio of the differences of values of the corresponding property or community metric for networks $c_{i}$ and $c_{j}$ to its maximum absolute value.

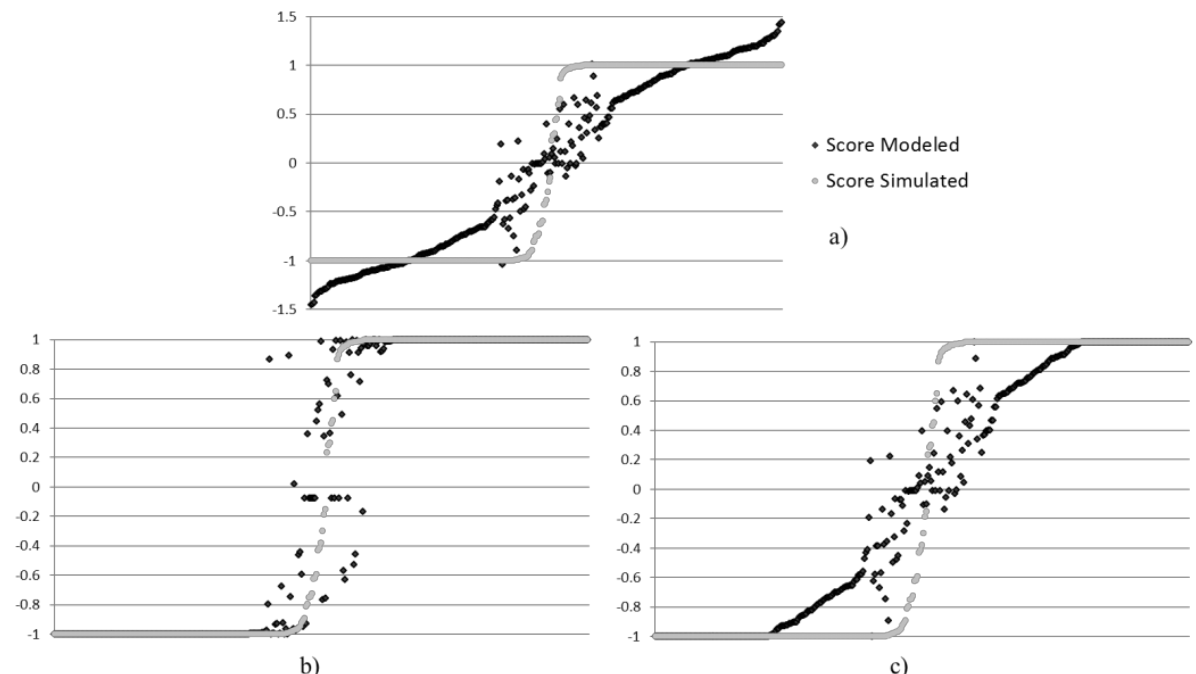

Fig. 2. Sample values of modeled score and simulated score values

We obtained linear models (lm's) using the software R [17] and the package leaps [18].. Fig 2. shows the pairs of values obtained from simulations compared with the ones obtained with the lm's. Fig. 2 a) shows the values of the variable Score and a sample $\mathrm{lm}$ output. One can observe that the value of the modeled variable goes out of the range $[-1,1]$. In such case, one option is to apply correction to the outputs out of the boundaries, as it is shown in Fig. 2 c), to bring them into the range. We will refer to the resulting model as the corrected $\mathrm{lm}$. Another option is to transform the variable Score to a saturated growth model, as represented in Equation (2); and then obtain the $\operatorname{lm}$ of the transformed variable $a$. In that case, it is important to avoid the numerical error of computing logarithm for argument 0 , thus, we rescaled outputs of the model into the range [-0.9999, 0.9999].

$$
\begin{gathered}
\text { score }=1-e^{a} \\
1-\text { score }=e^{a} \\
a=\left\{\begin{array}{c}
\ln (1+\text { score }), \text { score }<0 \\
-\ln (1-\text { score }), \text { score } \geq 0
\end{array}\right.
\end{gathered}
$$

These models are used to predict the scores of each network pairing, and we compute the mean absolute error (MAE), the mean squared error (MSE), and the Pearson correlation of the simulated scores with the modeled scores. 


\section{$5 \quad$ Results}

The first observation is that there are many games that did not converge with the $\Delta \mathrm{L}_{\mathrm{AB}}=100$. Thus, in the future work, we will use $\Delta \mathrm{L}_{\mathrm{AB}}$ proportional to the community size to avoid that effect of a contact number of links.

The several strategies produce small variation on outcomes over all games. Yet, if we consider the global outputs, the best strategy for the LF-NG is $x=-1$ while the worst is $x=1$; opposite is true in the case of SF-NG. Even though the results show that numbers of wins in each of the two NG variants are the same, the wins come from pairings between different networks in each case. Moreover, the outcome may also be affected by the quality metrics of network communities, with modularity density $\mathrm{Q}_{\mathrm{ds}}$, being the predictor. More discussion of this case is included in Section 5.2.

\subsection{Modeling Pairing Results}

Table 4 shows the results obtained from the models described in Section 4. We can observe that the saturated models have a lower mean absolute error, MAE, than the corrected-lm. However, for the mean squared error, MSE, there are cases when it is higher for the saturated model than for the corrected-lm; this happens when the saturated model fits correctly more points, but when it errs, the errors are usually high compared to the corrected-lm errors, as can be observed in Fig. 2 b and Fig. 2 c.

Table 4. Evaluation metrics of the obtained models. Rows MAE, MSE and Correlation show the values for the corrected $\mathrm{lm}$ and the saturated model.

$\begin{array}{lllll}\text { Game } & \text { MAE } & \text { MSE } & \text { Correlation } & \text { Lm } \\ \text { Speaker 0 0 } & 0.231 ; 0.0924 & 0.1205 ; 0.1057 & 0.9417 ; 0.9395 & \text { Eq (3) } \\ \text { Speaker 1 0 } & 0.3244 ; 0.1526 & 0.2025 ; 0.1384 & 0.8887 ; 0.9231 & \text { Eq(4) } \\ \text { Speaker -1 0 } & 0.197 ; 0.099 & 0.105 ; 0.1064 & 0.946 ; 0.944 ; & \text { Eq(5) } \\ \text { Speaker 1 -1 } & 0.334 ; 0.233 & 0.2275 ; 0.314 & 0.871 ; 0.832 & \text { Eq(6) } \\ \text { Speaker 1 1 } & 0.2339 ; 0.1225 & 0.1295 ; 0.1365 & 0.923 ; 0.9303 & \text { Eq(7) } \\ \text { Speaker -1 -1 } & 0.1329 ; 0.05158 & 0.0571 ; 0.0467 & 0.9696 ; 0.9729 & \text { Eq(8) } \\ \text { Listener 0 0 } & 0.1596 ; 0.078 & 0.0799 ; 0.099 & 0.935 ; 0.9429 & \text { Eq(9) } \\ \text { Listener 1 0 } & 0.2123 ; 0.0905 & 0.120 ; 0.0912 & 0.935 ; 0.9474 & \text { Eq(10) } \\ \text { Listener -1 0 } & 0.2074 ; 0.1152 & 0.1145 ; 0.1156 & 0.9389 ; 0.9369 & \text { Eq(11) } \\ \text { Listener 1 -1 } & 0.2425 ; 0.1622 & 0.1636 ; 0.1939 & 0.9109 ; 0.8946 & \text { Eq(12) } \\ \text { Listener 1 1 } & 0.205 ; 0.0580 ; & 0.1097 ; 0.05212 & 0.941 ; 0.969 & \text { Eq(13) } \\ \text { Listener -1 -1 } & 0.2291 ; 0.1252 ; & 0.1054 ; 0.1384 & 0.9465 ; 0.9234 & \text { Eq(14) }\end{array}$

In general, we can observe that in SF-NG, the networks with low community quality perform well; as indicated by their low or even negative modularity density $\mathrm{Q}_{\mathrm{ds}}$, large number of border-edges, BE, high conductance CND, expansion EXP, and the number of nodes, $|\mathrm{V}|$ (an exception is Equation (8)), but negatively correlated with contraction CNT.

The score equations for SF-NG are listed below. 


$$
\begin{aligned}
& \text { score }=-7.2510^{-4}+1.468|\mathrm{~V}|-0.852 Q d s-0.9093 d-0.892 H S C+0.2811 B E \\
& \text { score }=0.1065+1.14|\mathrm{~V}|-0.671 \mathrm{~s}+1.40 C N D-0.279 H S C-0.857 B D \\
& \text { score }=-0.0609+1.42|\mathrm{E}|-0.457 C N T+0.1366 r-0.233 H S C-0.8123 d \\
& \text { score }=0.1009+1.032|\mathrm{~V}|+0.5058|\mathrm{E}|-0.371 C N T-0.389 \mathrm{~s}+0.103 E X P \\
& \text { score }=-0.001158+1.64|\mathrm{~V}|-0.892|\mathrm{E}|+0.983|C|+0.68966 C G-0.6978 s \\
& \text { score }=-5.6410^{-4}-1.65|\mathrm{~V}|+2.373|\mathrm{E}|+0.585|C|-0.1748 C N T-0.242 d
\end{aligned}
$$

In case of LF-NG, we can observe that models correlate negatively with contraction CNT and the number of nodes $|\mathrm{V}|$ (with the exception of Equation (14).). At the same time, the models correlate positively with expansion EXP, conductance CND and the number of boarder edges, BE. This suggests that networks with low $\mathrm{Q}_{\mathrm{ds}}$ perform well. The score equations for LF-NG are listed below.

$$
\begin{aligned}
& \text { score }=5.3110^{-5}-1.818|\mathrm{~V}|+2.216|\mathrm{E}|+0.7939|C|+0.6381 \lambda-0.2589 \text { CNT } \\
& \text { score }=-0.00949-1.7685|\mathrm{~V}|+1.674|\mathrm{E}|+0.878|C|+0.95 \lambda+0.2188 \text { EXP } \\
& \text { score }=-0.01625+1.4|\mathrm{E}|-0.4479 C N T-0.6352 d+0.0926 r-0.170 H S C \\
& \text { score }=0.005394-1.315|\mathrm{~V}|+1.719|\mathrm{E}|+0.647|C|+0.6157 \lambda+0.3485 C N D \\
& \text { score }=-3.6210^{-4}-1.477|\mathrm{~V}|+1.212|\mathrm{E}|+0.7831|C|+1.265 \lambda+0.2234 B E \\
& \text { score }=-5.1510^{-4}+0.959|\mathrm{~V}|+0.335|\mathrm{E}|-0.586 s-0.335 H S C+0.448 E X P
\end{aligned}
$$

In Section 3.1, we observed that LF-NG with strategy $x=0$ is highly correlated with the number of edges $|E|$; while SF-NG with strategy $x=0$ is highly correlated with border-density BD. Our simulations show that for LF-NG with $\mathrm{x}=0$, Score $\mathrm{S}$ is highly correlated with the number of edges $|\mathrm{E}|$ and inversely correlated with the number of nodes, $|\mathrm{V}|$. In the case of SF-NG with $\mathrm{x}=0$, Score $\mathrm{S}$ it is directly proportional to $|\mathrm{V}|$ and seems that networks with lower community quality have an advantage.

\subsection{Selection of Communication Partners in NG}

In Section 4, we proposed a set of three strategies for selecting the second agent in each conversation. It is interesting question which strategy provides the best performance in various situations. To answer this question, we used the real social networks with known community structures: football [19], dolphin [20], karate [21] and Santafe [22]. We simulated each network paired with its copy using strategies $x=1$ and $x=-1$, (for example [football $\mathrm{x}=1$ ] vs. [football $\mathrm{x}=-1$ ]) with the addition of 100 links. Table 5 shows for each network which strategy is the best when each network is paired with its copy. These preliminary results suggest that the best strategy selection can be affected by the community structure quality of the networks, estimated with $\mathrm{Q}_{\mathrm{ds}}$.

Table 5. Performance of strategies $x=1$ and $x=-1$ in the game of two copies of the same network. A value of 1 indicates winning by $x=1$ strategy, while -1 indicates advantage of $x=-1$ strategy.

$\begin{array}{llrr} & Q_{\text {ds }} & \text { SF-NG } & \text { LF-NG } \\ \text { Football } & 0.4281 & 1 & -1 \\ \text { Karate } & 0.1828 & -1 & 1 \\ \text { Dolphin } & 0.1368 & -1 & 1 \\ \text { Santafe } & 0.1043 & -1 & 1\end{array}$


Table 6. Performance of strategy $x=1$ and $x=-1$ in the game of two copies of the same network. A value of 1 indicates advantage of $x=1$ strategy, while -1 indicates winning by $x=-1$ strategy; values of 0.5 or -0.5 indicate slight advantage of the corresponding strategy.

$\begin{array}{rrr}\mathbf{Q}_{\mathbf{d}} \mathbf{S} & \text { SF-NG } & \text { LF-NG } \\ 0.039 & -1 & 1 \\ 0.039 & -1 & 1 \\ 0.046 & -1 & 1 \\ 0.084 & -1 & 1 \\ 0.134 & -1 & 1 \\ 0.163 & -1 & 1 \\ 0.173 & -1 & 1 \\ 0.21 & -0.5 & -1 \\ 0.246 & 0.5 & -1 \\ 0.323 & 1 & -1 \\ 0.339 & 1 & -1 \\ 0.343 & 1 & -1 \\ 0.356 & 1 & -1 \\ 0.378 & 1 & -1 \\ 0.407 & 1 & -1 \\ 0.408 & 1 & -1 \\ 0.418 & 1 & -1 \\ 0.422 & 1 & -1 \\ 0.424 & 1 & -1 \\ 0.439 & 1 & -1 \\ 0.449 & 1 & -1 \\ 0.469 & 1 & -1 \\ 0.471 & 1 & -1\end{array}$

We tested the effect of increasing the number of links being added between communities until the NG converged and reached consensus on a single opinion. This avoided the annoying lack of convergence in many relatively poorly connected networks. The results are shown in Table 6; the conclusions implied by results in Table 6 are in agreement with the ones drawn on the basis of results shown in Table 5. Networks with low $\mathrm{Q}_{\mathrm{ds}}$ obtained better results with $\mathrm{x}=-1$ strategy for SF-NG and with $\mathrm{x}=1$ strategy for LF-NG. In contrast, networks with high $Q_{\mathrm{ds}}$ obtained better results with $\mathrm{x}=1$ strategy for SF-NG and with $\mathrm{x}=-1$ strategy for LF-NG. The results were obtained by treating $\mathrm{Q}_{\mathrm{ds}} \geq 0.343$ as high and $\mathrm{Q}_{\mathrm{ds}} \leq 0.173$ as low. Which strategy is the best for $\mathrm{Q}_{\mathrm{ds}}$ values between $(0.173,0.343)$ is not clear, and the answer may also depend on the type of game (SF-NG or LF-NG). In our results, we can observe that with $\mathrm{Q}_{\mathrm{ds}}=$ 0.246 , SF-NG with $x=1$ strategy has an advantage while with $Q_{d s}=0.21$, $x=-1$ strategy preforms better. Hence, the turning point is likely to be between these two values. We will further investigate the position of this turning point in future work.

The results also indicate that the behavior of each strategy depends on network structure as measured by parameters, such as $\mathrm{Q}_{\mathrm{ds}}$. This probably limits the precision of prediction that can be obtained with linear models. It should also be noted that the set of networks used contains more networks with high $\mathrm{Q}_{\mathrm{ds}}$ than with the low one; it may indicate why the best strategies on average were $x=-1$ for LF-NG and $x=1$ for SF-NG. 


\section{Conclusions}

It is important to notice that when we stage a NG, we modify the initial properties of the networks by adding new links that may introduce significant change to the community structures. The preliminary case study described here has shown that the type of NG and bias in selecting the speaker or listener influence to which opinion the two networks are likely to converge.

The main conclusion of this paper is that the quality of a network community structure is directly related to network's ability to resist or exert influence from/on others. We also observe that the modularity density $\mathrm{Q}_{\mathrm{ds}}$ is highly predictive of which network is most likely to impose its opinion for consensus in the merged networks. LF-NG scores are highly correlated with the number of edges $|\mathrm{E}|$ and highly inversely correlated with the number of nodes $|\mathrm{V}|$. In contrast, SF-NG scores are highly positively correlated with the number of nodes $|\mathrm{V}|$. Furthermore, when the two networks have similar sizes (both in terms of the numbers of edges $|\mathrm{E}|$ and nodes $|\mathrm{V}|$ ), the network with higher community structure quality performs poorly in SF-NG but well with LFNG.

Acknowledgments. This work was supported in part by the Army Research Laboratory under Cooperative Agreement Number W911NF-09-2-0053, by the Army Research Office Grant W911NF-12-1-0546, by the Office of Naval Research Grant No. N00014-09-1-0607, by the Polish National Science Centre, the decision no. DEC2013/09/B/ST6/02317, by the EU's 7FP under grant agreement no 316097, by the TIN2013-48040-R (QWAVES) Nuevos métodos de automatización de la búsqueda social basados en waves de preguntas, the IPT20120482430000 (MIDPOINT) Nuevos enfoques de preservación digital con mejor gestión de costes que garantizan su sostenibilidad, and VISUAL AD, RTC-2014-2566-7 and GEPID, RTC-2014-25767, as well as the grup de recerca consolidat CSI-ref. 2014 SGR 1469. The views and conclusions contained in this document are those of the authors and should not be interpreted as representing the official policies, either expressed or implied, of the Army Research Laboratory or the U.S. Government.

\section{References}

1. Schellings, T.C.: Micromotives and Macrobehavior. Norton (1978).

2. Castellano, C., Fortunato, S., Loreto, V.: Statistical physics of social dynamics. Rev. Mod. Phys. 81, 591-646 (2009).

3. Carr, J.: Applications of Centre Manifold Theory. Springer US (1982).

4. Clifford, P., Sudbury, A.: A model for spatial conflict. Biometrika. 60, 581-588 (1973).

5. Granovetter, M.: Threshold Models of Collective Behavior. Am. J. Sociol. 83, 1420-1443 (1978).

6. Bass, F.M.: A New Product Growth for Model Consumer Durables. Manage. Sci. 15, 215227 (1969).

7. Steels, L.: A self-organizing spatial vocabulary. Artif. Life. 2, 319-32 (1995).

8. Lu, Q., Korniss, G., Szymanski, B.K.: The Naming Game in social networks: community formation and consensus engineering. J. Econ. Interact. Coord. 4, 221-235 (2009). 
9. Dall'Asta, L., Baronchelli, A., Barrat, A., Loreto, V.: Nonequilibrium dynamics of language games on complex networks. Phys. Rev. E. 74, 036105 (2006).

10. Mazzitello, K.I., Candia, J., Dossetti, V.: Effects of Mass Media and Cultural Drift in a Model for Social Influence. Int. J. Mod. Phys. C. 18, 10 (2006).

11. Candia, J., Mazzitello, K.I.: Mass media influence spreading in social networks with community structure, (2008).

12. Mahadevan, P., Krioukov, D.: The Internet AS-level topology: three data sources and one definitive metric. ACM SIGCOMM. (2006).

13. Newman, M., Girvan, M.: Finding and evaluating community structure in networks. Phys. Rev. E. 69, 026113 (2004).

14. Chen, M., Nguyen, T., Szymanski, B.: A new metric for quality of network community structure. ASE Hum. 2, 226-240 (2013).

15. Suchecki, K., Holyst, J.: Bistable-monostable transitoin in the Ising model on two connected coplex networks, Physical Review E, 031110 (2009)

16. Chen, M., Kuzmin, K., Szymanski, B.: Community Detection via Maximization of Modularity and Its Variants. IEEE Trans. Comput. Soc. Syst. 1, 46-65 (2014).

17. R Core Team: R: A Language and Environment for Statistical Computing, http://www.rproject.org/, (2013).

18. Lumley, T.: leaps: regression subset selection, http://cran.r-project.org/package=leaps, (2009).

19. Girvan, M., Newman, M.E.J.: Community structure in social and biological networks. Proc. Natl. Acad. Sci. U. S. A. 99, 7821-6 (2002).

20. Lusseau, D.: The emergent properties of a dolphin social network. Proc. R. Soc. London. Ser. B Biol. Sci. 270, S186-S188 (2003).

21. Zachary, W.: An information flow model for conflict and fission in small groups. J. Anthropol. Res. 33, 452-473 (1977).

22. Newman, M.E.: The structure of scientific collaboration networks. Proc. Natl. Acad. Sci. U. S. A. 98, 404-9 (2001). 